\title{
On the Hydrodynamic Limit of the Enskog Equation*
}

\author{
By
}

\author{
Mirosław LACHOWICZ ${ }^{\dagger}$
}

\begin{abstract}
The Enskog equation in the hydrodynamic limit is investigated. If the Knudsen number $\varepsilon$ and the scale of diameter $a$ of the hard sphere particles are of the same order. the resulting system of hydrodynamic equations (the Enskog-Euler system) is different from that for the Boltzmann equation. The existence and uniqueness theorem, in this case for the Enskog equation, is proven on the time interval independent of the small parameters. As $\varepsilon \downarrow 0$, the solution of the Enskog equation tends to the Maxwellian whose fluid-dynamic parameters solve the Enskog-Euler system. As a by-product - the existence theorem for the Enskog-Euler system is obtained.
\end{abstract}

Key word. Enskog equation, Euler system, singularly perturbed problem, hydrodynamic limit.

\section{$\S 1$. Introduction}

In the Boltzmann equation, which is a model of kinetic theory. the overall dimensions of the particles are neglected (cf. [8] and [5]). In the case of dense gases, however, one should replace this mass-point model by a model which can take into account the overall dimensions of particles.

One such attempt leads to the Enskog equation - a quite successful model of kinetic theory of moderately dense gases (cf. $[8,4]$ and $[1,2,7]$ ), in which each particle is assumed to be a hard sphere with a nonzero diameter.

The Enskog equation, in the dimensionless form (see [10]), reads

$$
D f=\frac{1}{\varepsilon} E_{\varepsilon, a}(f ; f, f),
$$

where $D$ is the free-streaming operator

Communicated by H. Okamoto, February 12, 1997.

1991 Mathematics Subject Classification (s): 82C40, 45K05, 76P05.

* This work was partially supported by the Polısh State Committee for Scientific Research under the Grant 2P03A02311.

† Department of Mathematics, Warsaw Unıversity, ul. Banacha 2, PL-02097 Warsaw, Poland e-mall : lachowic@mimuw.edu.pl. 


$$
D=\frac{\partial}{\partial t}+\mathrm{v} \cdot \frac{\partial}{\partial \mathrm{x}}
$$

$t, \mathbb{x}$ and $\mathbb{v}$ are, respectively, the time, the position, and the velocity variables; $f=f(t, \mathbb{R}, \mathbb{v})$ is the one-particle distribution function ; $\varepsilon$ is the Knudsen number ; $a$ is the scale of diameter of the hard sphere; $E_{\varepsilon, a}$ is the Enskog collision operator

$$
\begin{aligned}
& E_{\varepsilon, a}(f ; f, f)(t, \mathbb{x}, \mathbb{v})=\int_{\mathbb{R}^{3}} \int_{\mathbb{S}^{2}}\left(\mathscr{Y}_{\varepsilon, a}(f ; t, \mathbb{\mathbb { x }}, \mathbb{\mathbb { x }}+a \mathbb{\mathbb { R }}) f\left(t, \mathbb{\mathbb { X }}+a \mathbb{\mathbb { R }}, \mathbb{W}^{\prime}\right) f\left(t, \mathbb{\mathbb { N }}, \mathbb{V}^{\prime}\right)\right. \\
& \left.-\mathscr{Y}_{\varepsilon, a}(f ; t, \mathbb{X}, \mathbb{Z}-a \mathbb{R}) f(t, \mathbb{X}-a \mathbb{R}, \mathbb{W}) f(t, \mathbb{X}, \mathbb{v})\right)(\mathbb{I} \circ(\mathbb{W}-\mathbb{V}) \vee 0) \mathrm{d} \mathbb{R} \mathbb{W} \mathbb{W}
\end{aligned}
$$

The standard notation is used - a particle with the center at $x$ and the velocity $\mathbb{v}$ collides with a particle with the center at $\mathbb{X}-a \mathbb{R}$ and the velocity $\mathbb{W}$;

$$
\mathbb{S}^{3}=\left\{\mathbb{n} \in \mathbb{R}^{3}:|\mathbb{R}|=1\right\} ;
$$

$\mathbb{v}^{\prime}$ and $\mathbb{w}^{\prime}$ are functions of $\mathbb{v}$ and $\mathbb{w}$ as well as on $\mathbb{m} \in \mathbb{S}^{3}$ according to

$$
\begin{aligned}
\mathbb{w}^{\prime} & =\mathbb{v}+(\mathbb{R} \cdot(\mathbb{w}-\mathbb{v})) \mathfrak{m}, \\
\mathbb{w}^{\prime} & =\mathbb{W}-(\mathbb{R} \cdot(\mathbb{w}-\mathbb{v})) \mathfrak{R} .
\end{aligned}
$$

The collision kernel corresponds to the hard sphere model; $\alpha_{1} \vee \alpha_{2}$ means the maximum of the two numbers $\alpha_{1}$ and $\alpha_{2}$ (whereas $\alpha_{1} \wedge \alpha_{2}=\min \left(\alpha_{1}, \alpha_{2}\right)$ ).

$\mathscr{Y}_{\varepsilon, a}$ represents the pair correlation function. The different ways in which one models the pair correlation function give rise to the different kinetic Enskog equations found in the literature (see [4]).

The present paper considers only the simplified case of $\mathscr{Y}_{\varepsilon, a} \equiv 1$, in the literature referred to as the Boltzmann-Enskog equation. However, the analysis is also valid for a general case, under a suitable assumption about the behaviour of the factor $\mathscr{Y}_{\varepsilon, a}$ (cf. [10]).

The mathematical theory for the Enskog equation can be found in $[1,2,4$, 7] (see also references therein).

It is very well-known (cf. $[8,10]$ ) that the hydrodynamic limit of the Enskog equation (1.1) in the regime $a \sim \varepsilon \downarrow 0$ should be different from that in the regime $a \ll \varepsilon \downarrow 0$ (e.g. for $a \sim \varepsilon^{q}$ with $q>1$ ). In the latter case the Enskog equation results in the classical system for compressible fluid (see [10]), exactly like the Boltzmann equation, whereas in the former, "Enskog terms" appear in the resulting hydrodynamic equations.

The present paper is a first step towards a rigorous description of the hydrodynamic limit when the Enskog effects are not negligible.

Introduce a new dimensionless parameter $b>0$ such that

$$
a=b \varepsilon .
$$

In this paper the parameter $b$ is assumed to be fixed in the (hydrodynamic) 
limit $\varepsilon \downarrow 0$, but not greater than some critical constant (independent of $\varepsilon$ ) -cf. (3.26a). Such a smallness assumption admits of a clear physical interpretation.

The Paper is organized in the following way. In $\S 2$ the formal expansion procedure is described, and the (Enskog-Euler) system of hydrodynamic equations is defined. $\S 3$ presents mathematical preliminaries and establishes the existence and uniqueness of classical solutions (for initial data close to a global equlibrium) for the Enskog equation, on the time interval $\left[0, t_{0}\right]$, where $t_{0}$ does not depend on $\varepsilon$ (i.e. the time interval has a "macroscopic" character). In $\S 4$ the main convergence result is stated and proved - the solutions of the Enskog equation tend to the Maxwellian whose fluid-dynamic parameters solve the Enskog-Euler system of hydrodynamic equations. As a by-product, the existence (however without uniqueness) of a classical solution for the EnskogEuler system is obtained. The present result is the first (according to the author's knowledge) existence result for it (cf. Remark 4.1).

Throughout the paper, the small letter $c$ is reserved for a positive constant, independent of all the relevant variables and parameters. Appearing of the letter $c$, with or without subscripts, in a formula, is understood that the formula is valid for some constant $c>0$.

\section{§. Formal Expansion Procedure}

Consider the following singularly perturbed problem for the BoltzmannEnskog equation

$$
\begin{gathered}
D f=\frac{1}{\varepsilon} J_{\varepsilon}(f, f), \\
\left.f\right|_{t=0}=F,
\end{gathered}
$$

where $\varepsilon>0$ is a small parameter ;

$$
\begin{aligned}
J_{\varepsilon}\left(f_{\mathbf{1}}, f_{2}\right) & =\frac{1}{2}\left(J_{\varepsilon}^{+}\left(f_{1}, f_{2}\right)+J_{\varepsilon}^{+}\left(f_{2}, f_{1}\right)-f_{1} \nu_{\varepsilon}\left(f_{2}\right)-f_{2} \nu_{\varepsilon}\left(f_{\mathbf{1}}\right)\right), \\
J_{\varepsilon}^{+}\left(f_{1}, f_{2}\right)(t, \mathbf{x}, \mathbf{v}) & =\int_{\mathbb{R}^{3}} \int_{\mathbb{S}^{2}} f_{\mathbf{1}}\left(t, \mathbf{x}+b \varepsilon \mathbf{n}, \mathbf{w}^{\prime}\right) f_{2}\left(t, \mathbf{x}, \mathbf{w}^{\prime}\right)(\mathbf{n} \cdot(\mathbf{w}-\mathbf{v}) \vee 0) \mathrm{d} \mathbf{n} \mathrm{d} \mathbf{w}, \\
\nu_{\varepsilon}(f)(t, \mathbf{x}, \mathbf{v}) & =\int_{\mathbb{R}^{3}} \int_{\mathbb{S}^{2}} f(t, \mathbf{x}-b \varepsilon \mathbf{n}, \mathbf{w})(\mathbf{n} \cdot(\mathbf{w}-\mathbf{v}) \vee 0) \mathrm{d} \mathbf{n d} \mathbf{w}
\end{aligned}
$$

and the scale of the diameter $a$ is related to the parameter $b$ by (1.2).

The parameter $b$ is assumed to be a fixed positive number.

According to the theory of perturbed problems, the behaviour of the solutions of Problem (2.1), in the hydrodynamic limit $\varepsilon \downarrow 0$, can be studied by comparing a solution of (2.1) with a solution of the corresponding degenerate problem, which is obtained by formally putting in (2.1a) the small parameter $\varepsilon$ equal to 0 . This leads to 


$$
J_{0}(f, f)=0
$$

where $J_{0}=\left.J_{\varepsilon}\right|_{\varepsilon=0}$ is the classical Boltzmann collision operator.

A unique class of solutions of Eq. (2.2) is that of Maxwellians

$$
M[\rho, \mathbb{Z}, T](t, \mathbb{\mathbb { X }}, \mathbb{v})=\rho(t, \mathbb{\mathbb { x }})(2 \pi T(t, \mathbb{\mathbb { X }}))^{-3 / 2} \exp \left(-\frac{|\mathbb{v}-\mathbb{\sharp}(t, \mathbb{\mathbb { X }})|^{2}}{2 T(t, \mathbb{X})}\right),
$$

where $\rho$ (local density), 如 (macroscopic velocity vector), and $T$ (macroscopic temperature) are the fluid-dynamic parameters of the Maxwellian $M=M[\rho$, $\mathbb{U}$, $T]$.

One can expect that in the limit $\varepsilon \downarrow 0$ the solution of Eq. (2.1a) should tend to the Maxwellian $M[\rho, \mathbb{u}, T]$, whose fluid-dynamic parameters solve a system of equations of continuum theory.

Assume that $\rho$, ㅈ, $T$ are known, smooth, and that $\rho$ together with $T$ are positive. For fixed $t$ and $x$ consider the space $L_{2}\{M\}$ equipped with the norm $\left\|f ; L_{2}\{M\}\right\|=\left\|f M^{-1 / 2} ; L_{2}\left(\mathbb{R}^{3}\right)\right\|$, and with the inner product $\left(f_{1}, f_{2}\right)_{L_{2}\{M\}}=\left(f_{1} M^{-1}\right.$, $\left.f_{2}\right)_{L_{2}\left(\mathbb{R}^{3}\right)}$, where $\left\|\circ ; L_{2}\left(\mathbb{R}^{3}\right)\right\|$ and $(\circ,)^{\circ} L_{L_{2}\left(\mathbb{R}^{3}\right)}$ are the norm and the inner product, respectively, in the space $L_{2}\left(\mathbb{R}^{3}\right)$.

Define the "hydrodynamic" and "nonhydrodynamic" subsets in $L_{2}\{M\}$

$$
\mathcal{N}=\operatorname{lin}\left\{M \phi_{1}: l=0, \ldots, 4\right\},
$$

and

$$
\mathscr{R}=\mathcal{N}^{\perp}=\left\{f \in L_{2}\{M\}:\left(f, M \psi_{l}\right)_{L_{2}\{M\}}=0 ; l=0, \ldots, 4\right\},
$$

where $\phi_{0}, \ldots, \phi_{4}$ are the collision invariants

$$
\phi_{0} \equiv 1, \phi_{l}(\mathbb{v})=v_{l} \quad(l=1,2,3), \phi_{4}(\mathbb{v})=|\mathbb{v}|^{2},
$$

and $v_{l}$ is the $l$-th component of the vector $\nabla$.

Define in $L_{2}\{M\}$ the orthogonal projection operators $\mathscr{P}$ and $\mathscr{P} \perp=1-\mathscr{P}$ onto $\mathcal{N}$ and $\mathscr{R}$, respectively.

In the Hilbert procedure, although Problem (2.1) is singularly perturbed in the limit $\varepsilon \downarrow 0$, the solution is searched in the regular form

$$
f=\sum_{j=0} \varepsilon^{j} f^{(j)},
$$

where $f^{(j)}=g^{(j)}+h^{(j)}$ for $j \geq 1$ and

$$
g^{(j)} \in \mathscr{R} \text { and } h^{(j)} \in \mathcal{N} .
$$

This leads to the following set of equations

$$
\begin{gathered}
J_{0}\left(f^{(0)}, f^{(0)}\right)=0 ; \\
2 J_{0}\left(f^{(0)}, g^{(1)}\right)=\mathscr{P}^{\perp} D f^{(0)}-b \mathscr{P}^{\perp} J_{0}^{(1)}\left(f^{(0)}, f^{(0)}\right) ;
\end{gathered}
$$




$$
\mathscr{P} D f^{(0)}-b \mathscr{P} J_{0}^{(1)}\left(f^{(0)}, f^{(0)}\right)=0
$$

where

$$
\begin{aligned}
J_{0}^{(l)}\left(f_{1}, f_{2}\right) & =\frac{1}{2}\left(J_{0}^{(l)+}\left(f_{1}, f_{2}\right)+J_{0}^{(l)+}\left(f_{2}, f_{1}\right)-f_{1} \nu_{0}^{(l)}\left(f_{2}\right)-f_{2} \nu_{0}^{(l)}\left(f_{1}\right)\right), \\
J_{0}^{(l)+}\left(f_{1}, f_{2}\right)(t, \mathbf{x}, \mathbf{v}) & =\frac{1}{l !} \int_{\mathbb{R}^{3}} \int_{\mathbb{S}^{2}}\left(\left(\mathbf{n} \cdot \frac{\partial}{\partial \mathbf{x}}\right)^{l} f_{1}\right)\left(t, \mathbf{x}, \mathbf{w}^{\prime}\right) f_{2}\left(t, \mathbf{x}, \mathbf{v}^{\prime}\right)(\mathbf{n} \cdot(\mathbf{w}-\mathbf{v}) \vee 0) \mathrm{d} \mathbf{n d} \mathbf{w}, \\
\nu_{0}^{(l)}(f)(t, \mathbf{x}, \mathbf{v}) & =\frac{1}{l !} \int_{\mathbb{R}^{3}} \int_{\mathbb{S}^{2}}\left(\left(-\mathbf{n} \cdot \frac{\partial}{\partial \mathbf{x}}\right)^{l} f\right)(t, \mathbf{x}, \mathbf{w})(\mathbf{n} \cdot(\mathbf{w}-\mathbf{v}) \vee 0) \mathrm{d} \mathbf{n d w} .
\end{aligned}
$$

Equation (2.5) yields

$$
f^{(0)}=M[\rho, \mathbb{Q}, T],
$$

for some parameters $\rho$, и, $T$.

Hence, System (2.7) assumes the form (cf. [8])

$$
\begin{gathered}
\frac{\partial}{\partial t} \rho+\sum_{l=1}^{3} \frac{\partial}{\partial x_{l}}\left(\rho u_{l}\right)=0 ; \\
\frac{\partial}{\partial t}\left(\rho u_{j}\right)+\sum_{l=1}^{3} \frac{\partial}{\partial x_{l}}\left(\rho u_{l}\right)+\frac{\partial}{\partial x_{j}}\left(\rho T\left(1+\frac{2}{3} \pi b \rho\right)\right)=0, \quad j=1,2,3 ; \\
\frac{\partial}{\partial t}\left(\rho\left(\frac{3}{2} T+\frac{1}{2}|\mathbf{u}|^{2}\right)\right)+\sum_{l=1}^{3} \frac{\partial}{\partial x_{l}}\left(\rho u_{l}\left(\frac{3}{2} T+\frac{1}{2}|\mathbf{u}|^{2}\right)+\rho u_{l} T\left(1+\frac{2}{3} \pi b \rho\right)\right)=0 .
\end{gathered}
$$

System (2.9) is referred to, in the present paper, as the Enskog-Euler system. Note that if one set $b=0$ it becomes the classical system of Euler equations for compressible fluid.

The justification of the Hilbert procedure for the Enskog equation, in the case of $b \sim$ const as $\varepsilon \downarrow 0$, still remains an open problem. In fact, the convergence rate which can be found by the methods of the present paper (as well as those of [14] for the Boltzmann equation) is not sufficiently strong for such a justification. On the other hand, the justification was proved for $b=\mathfrak{O}\left(\varepsilon^{q}\right)\left(q>q_{0}\right.$, for some $q_{0}>1$ ) -see [10].

Remark 2.1. Let $\widetilde{\mathfrak{b}}: \mathbb{S}^{2} \times \mathbb{R}^{3} \rightarrow \mathbb{R}_{+}^{1}$ be continuous function such that

$$
\begin{aligned}
& \widetilde{\mathfrak{b}}(\mathbf{n}, \mathbf{w}-\mathbf{v})=\widetilde{\mathfrak{b}}(\mathbf{n}, \mathbf{v}-\mathbf{w})=\widetilde{\mathfrak{b}}\left(\mathbf{n}, \mathbf{w}^{\prime}-\mathbf{v}^{\prime}\right)=\widetilde{\mathfrak{b}}\left(\mathbf{n}, \mathbf{w}^{\prime}-\mathbf{w}^{\prime}\right)=\widetilde{\mathfrak{b}}(-\mathbf{n}, \mathbf{w}-\mathbf{v}) \\
& \forall \mathbf{n} \in \mathbb{S}^{2}, \forall \mathbf{v}, \mathbf{w} \in \mathbb{R}^{3} .
\end{aligned}
$$


Moreover, let $\widetilde{\mathfrak{b}}(\mathfrak{m}, \mathbb{w}-\mathbb{v})=\chi(\mathbb{m} \cdot(\mathbb{w}-\mathbb{v}) \geq 0) \widetilde{\mathfrak{b}}(\mathbb{m}, \mathbb{w}-\mathbb{v})$ satisfy Grad's cutoff hard potential assumption $([9]-(55))$. Consider Eq. (2.1) with the general collision kernel $\mathfrak{b}(\mathbb{n}, \mathbb{w}-\mathbb{v})$ instead of the hard sphere kernel $(\mathfrak{m} \circ(\mathbb{W}-\mathbb{V}) \vee$ $0)$ ). Under Assumption (1.2), such an equation results in the following system

$$
\begin{gathered}
\frac{\partial}{\partial t} \rho+\sum_{l=1}^{3} \frac{\partial}{\partial x_{l}}\left(\rho u_{l}\right)=0 ; \\
\frac{\partial}{\partial t}\left(\rho u_{l}\right)+\sum_{l=1}^{3} \frac{\partial}{\partial x_{l}}\left(\rho u_{l} u_{l}\right)+\frac{\partial}{\partial x_{j}}(\rho T)+b \sum_{l=1}^{3} \frac{\partial}{\partial x_{l}}\left(\rho^{2} \mathfrak{p}_{l j}(T)\right)=0, \quad j=1,2,3 ; \\
\frac{\partial}{\partial t}\left(\rho\left(\frac{3}{2} T+\frac{1}{2}|\mathbb{u}|^{2}\right)\right)+\sum_{l=1}^{3} \frac{\partial}{\partial x_{l}}\left(\rho u_{l}\left(\frac{3}{2} T+\frac{1}{2}|\mathfrak{u}|^{2}\right)+\rho u_{l} T\right)+b \sum_{l, j=1}^{3} \frac{\partial}{\partial x_{l}}\left(\rho^{2} u_{j} \mathfrak{p}_{l j}(T)\right)=0 ;
\end{gathered}
$$

where

$$
\begin{aligned}
& \mathfrak{p}_{l j}(T)= \\
& \frac{\sqrt{2 T}}{2 \pi^{3}} \iint_{\mathbb{R}^{2}} \int_{\mathbb{R}^{3}} n_{\mathbb{S}^{2}} m_{j}\left(\mathbb{\mathbb { R } ^ { \circ }}(\mathbb{w}-\mathbb{v}) \vee 0\right) \mathfrak{b}(\mathbb{R}, \sqrt{2 T}(\mathbb{w}-\mathbb{v})) \exp \left(-|\mathbb{v}|^{2}-\mid \mathbb{w}^{2}\right) \mathrm{d} \mathbb{m} d \mathbb{w} \mathrm{d} \mathbb{v} .
\end{aligned}
$$

Remark 2.2. The Enskog equation with the symmetrized kernel $|\mathfrak{n} \cdot(\mathbb{w}-\mathbb{v})|$ instead of $\mathbb{n}^{\circ}(\mathbb{W}-\mathbb{v}) \vee 0$ (or more generaly with the one $\widetilde{b}$ as in $(2.10))$, results in a different type of hydrodynamic equations. In fact, for $b \sim c$ (i.e. $a \sim c \varepsilon$ ) it results in the classical Euler system $([10])$, whereas for $b \sim \frac{c}{\sqrt{\varepsilon}}$ (i.e. $a \sim c \sqrt{\varepsilon})$ - in the Navier-Stokes-type system at the 0 -th order approximation (see [11]). The viscosity and heat conduction terms, in the latter system, are not $0(1)$ as $\varepsilon \downarrow 0$.

\section{§ 3. 世xistence of Soluritions}

The existence and uniqueness of solutions as well as the convergence for $\varepsilon \downarrow 0$ is proved by applying the concept of Ukai and Asano [14].

Throughout the paper $\omega$ is a global Maxwellian, i.e.

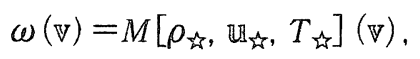

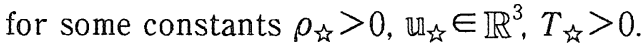

The initial datum is assumed in the form

$$
F=\omega+\omega^{\frac{1}{2}} G .
$$

where $G$ is independent of $\varepsilon$, and the solution to (2.1) is looked for in the form 


$$
f=\omega+\omega^{\frac{1}{2}} g .
$$

In terms of $g$ Problem (2.1) reads

$$
\begin{gathered}
\frac{\partial g}{\partial t}=-\mathrm{v} \cdot \frac{\partial g}{\partial \mathrm{x}}+\frac{1}{\varepsilon} L g+\frac{1}{\varepsilon} Q_{\varepsilon} g+\frac{1}{\varepsilon} \Gamma_{\varepsilon}(g, g), \\
\left.g\right|_{t=0}=G,
\end{gathered}
$$

where

$$
\begin{gathered}
L g=2 \omega^{-\frac{1}{2}} J_{0}\left(\omega, \omega^{\frac{1}{2}} g\right), \\
Q_{\varepsilon} g=2 \omega^{-\frac{1}{2}} J_{\varepsilon}\left(\omega, \omega^{\frac{1}{2}} g\right)-L g, \\
\Gamma_{\varepsilon}\left(g_{1}, g_{2}\right)=\omega^{-\frac{1}{2}} J_{\varepsilon}\left(\omega^{\frac{1}{2}} g_{1}, \omega^{\frac{1}{2}} g_{2}\right),
\end{gathered}
$$

(note that $J_{\varepsilon}(\omega, \omega)=0$ ).

Throughout the paper, for simplicity of notation, we continue not to indicate the $\varepsilon$-dependence of the functions, where no confusion can arise.

Consider the following integral version of Problem (3.2)

$$
g(t)=e^{t B_{\varepsilon}} G+\frac{1}{\varepsilon} \int_{0}^{t} e^{\left(t-t_{1}\right) B_{\varepsilon}}\left(Q_{\varepsilon} g\left(t_{1}\right)+\Gamma_{\varepsilon}(g, g)\left(t_{1}\right)\right) d t_{1}
$$

where $e^{t B_{\varepsilon}}$ denotes the semigroup (see $[6]$ and $[13,14]$ ) generated by the linear operator

$$
B_{\varepsilon}=-\mathbf{v} \cdot \frac{\partial}{\partial \mathbf{x}}+\frac{1}{\varepsilon} L
$$

In the present paper. Problem (3.3) is considered for $t>0, \mathbf{x} \in \mathbb{R}^{3}$ and $\mathbb{v} \in \mathbb{R}^{3}$. However, the proofs are applicable to the case $t>0, \mathbf{x} \in \mathbb{T}^{3}$ and $\mathbb{v} \in \mathbb{R}^{3}$, where $\mathbb{T}^{3}$ is the three-dimensional torus (this case corresponds to the rectangular domain with the specular reflection boundary condition), with the modifications given in [15] and [13].

The operator $L$ can be split into "regular" and "singular" parts ([9])

$$
L g=K g-\nu g,
$$

where

$$
\nu(\mathbb{v})=\left.\nu_{\varepsilon}(\omega)(\mathbb{v})\right|_{\varepsilon=0}=\iint_{\mathbb{R}^{3}} \omega(\mathbb{w})(\mathbb{N} \cdot(\mathbb{w}-\mathbf{v}) \vee 0) \mathrm{d} \mathbf{n d} \mathbf{w}
$$

is such that

$$
c_{-}(1+|v|) \leq \nu(\mathbb{v}) \leq c_{+}(1+|v|)
$$


The Banach spaces used in [14] (see also [13]) are needed. Let $\mathbb{B}^{\alpha}$ be the space equipped with the norm

$$
\|g\|^{(\alpha)}=\sup _{\nabla \in \mathbb{R}^{3}}\left|(1+|\mathbb{v}|)^{\alpha} g(\mathbb{v})\right|
$$

where $\alpha \in \mathbb{R}^{1}$.

Let $\widehat{g}=\mathscr{F} g$ denote the Fourier transform of a function $g \in \mathscr{S}^{\prime}\left(\mathbb{R}^{3} \times \mathbb{R}^{3}\right)$ with respect to the position variable $\mathbb{K}$,

$$
\widehat{g}(\mathbb{k}, \mathbb{v})=\mathscr{F} g(\mathbb{R}, \mathbb{v})=\frac{1}{(2 \pi)^{3 / 2}} \int_{\mathbb{R}^{3}} e^{-\imath \mathbb{k} \cdot \mathbb{s}} g(\mathbb{x}, \mathbb{v}) \mathrm{dx}, \quad \mathbb{k} \in \mathbb{R}^{3}, \quad i=\sqrt{-1} .
$$

Let $\mathbb{X}_{\beta, \gamma}^{(\alpha)}$ denote the space equipped with the norm

$$
\|g\|_{\beta, \gamma}^{(\alpha)}=\sup _{\mathbf{k}, \boldsymbol{v} \in \mathbb{R}^{3}}\left|(1+|\mathbb{v}|)^{\alpha}(1+|\mathbb{K}|)^{\beta} \exp (\gamma|\mathbb{k}|) \widehat{g}(\mathbb{K}, v)\right|,
$$

where $\alpha, \beta, \gamma \in \mathbb{R}_{+}^{1}$.

The space $\dot{\mathbb{X}}_{\beta, r}^{(\alpha)}$ is the closed subspace of $\mathbb{X}_{\beta, r}^{(\alpha)}$ such that

$$
g \in \dot{X}_{\beta, \gamma}^{(\alpha)} \Leftrightarrow g \in \mathbb{X}_{\beta, \gamma}^{(\alpha)} \quad \& \quad\left\|\mathscr{F}^{-1}\left(\chi\left(\left|\mathbb{R}_{\mathbb{R}}\right|+|\mathbb{v}|>\xi\right) \widehat{g}(\mathbb{K}, \mathbb{v})\right)\right\|_{\beta, \gamma}^{(\alpha)} \rightarrow 0, \quad \text { as } \quad \xi \rightarrow \infty \text {, }
$$

where $\chi(|\mathbb{k}|+|\mathbb{N}|>\xi)$ is the characteristic function of the domain

$$
\left\{(\mathbb{R}, \mathbb{v}) \in \mathbb{R}^{3} \times \mathbb{R}^{3}:|\mathbb{R}|+|\mathbb{v}|>\xi\right\} .
$$

Then let

$$
\mathbb{Y}_{\beta, \gamma}^{\alpha, \eta}(I)=\left\{g=g(t): \widetilde{g}_{n} \in C_{B}^{0}\left(I ; \dot{\mathbb{X}}_{\beta, \gamma}^{(\alpha)}\right) ; \widetilde{g}_{\eta}(t, \mathbb{X}, \mathbb{v}) \equiv \mathscr{F}^{-1} \exp (-\eta t|\mathbb{R}|) \widehat{g}(t, \mathbb{K}, \mathbb{\forall})\right\}
$$

be the space equipped with the norm

$$
\|g\|_{\beta, r, I}^{\alpha, \eta}=\sup _{t \in I}\|g(t)\|_{\beta, \gamma-\eta t}^{(\alpha)},
$$

for $\eta \geq 0$ and an interval $I \subset \mathbb{R}^{1}$, where $C_{B}^{0}(I ; \mathbb{X})$ denotes the space of $\mathbb{X}$-valued continuous bounded functions on $I$.

Finally the Banach space of $\varepsilon$-dependent functions is introduced

$$
\left.\left.\mathbb{Z}_{\beta, r, t_{0}}^{\alpha, n, 1}=C_{B}^{0}(] 0,1\right] ; \mathbb{Y}_{\beta, r}^{\alpha, \eta}\left(\left[0, t_{0}\right]\right)\right),
$$

with the norm

$$
\|g\|_{\beta, r, t_{0}}^{\alpha, \eta, 1}=\sup _{\substack{0<\varepsilon \leq 1 \\ 0 \leq t \leq t_{0}}}\|g(t)\|_{\beta, r-\eta t}^{(\alpha)}
$$

For simplicity of notation, the parameters $\alpha, \beta, \gamma, \eta, t_{0}$, are assumed to be properly chosen (cf. $(3.25-26)$ ) and fixed, and the spaces as well as the norms are denoted 


$$
\begin{gathered}
\mathbb{X}_{\beta, r}^{(\alpha)} \equiv \mathbb{X} ; \quad\|\cdot\|_{\mathbb{X}} \equiv\|\cdot\|_{\beta, r}^{(\alpha)} ; \\
\dot{\mathbb{X}} \equiv \dot{\mathbb{X}}_{\beta, r}^{(\alpha)} \\
\mathbb{Y}(I) \equiv \mathbb{Y}_{\beta, \gamma}^{\alpha, \eta}(I) ; \quad\|\cdot\|_{\mathbb{Y}(I)} \equiv\|\cdot\|_{\beta, r, I}^{\alpha, \eta} ;
\end{gathered}
$$

and

$$
\mathbb{Z} \equiv \mathbb{Z}_{\beta, r, t_{0}}^{\alpha, n, 1}: \quad\|\cdot\|_{\mathbb{Z}} \equiv\|\cdot\|_{\beta, r, t_{0} .}^{\alpha, n, 1}
$$

Define

$$
\widehat{B}_{\varepsilon}(\mathbf{k}) \widehat{g}(\mathbf{k}, \mathbf{v}) \equiv \mathscr{F}_{B_{\varepsilon}} g(\mathbf{k}, \mathbf{v}) \equiv\left(-i \mathbf{k} \cdot \mathbf{v}+\frac{1}{\varepsilon} L\right) \widehat{g}(\mathbf{k}, \mathbf{v}) .
$$

For each fixed $\varepsilon>0$ the semigroup $e^{t \hat{B}_{\varepsilon}(\mathbf{k})}$ is such that $([6,13,14])$

$$
e^{t \hat{B}_{\varepsilon}(\mathbf{k})}=\chi(\varepsilon|\mathbf{k}| \leq \kappa) \sum_{j=0}^{4} e^{\lambda_{j}^{\xi}(\mathbf{k}) t} P_{j}^{\varepsilon}(\mathbf{k})+U_{\varepsilon}(t, \mathbf{k}),
$$

where $\chi(\varepsilon|\mathbb{k}| \leq \kappa)$ is the characteristic function of the domain $\left\{\mathbb{R} \in \mathbb{R}^{3}: \varepsilon|\mathbb{k}| \leq \kappa\right\}$; $\kappa$ is a positive constant; $\lambda_{j}^{\varepsilon} \in C^{\infty}([-\kappa, \kappa]) \quad($ for $j=0, \ldots, 4)$ are such that $\Re \lambda_{j}^{\varepsilon}(\mathbf{k}) \leq 0$ and have the asymptotic expansion

$$
\lambda_{j}^{\varepsilon}(\mathbf{k})=i \lambda_{j}^{(1)}|\mathbf{k}|-\varepsilon \lambda_{j}^{(2)}|\mathbf{k}|^{2}+\mathscr{O}\left(\varepsilon^{2}|\mathbf{k}|^{3}\right), \quad|\mathbf{k}| \rightarrow 0,
$$

with coefficients $\lambda_{j}^{(1)} \in \mathbb{R}^{1}$ and $\lambda_{j}^{(2)}>0$;

$$
P_{j}^{\varepsilon}(\mathbf{k})=P_{j}^{(0)}\left(\frac{\mathrm{k}}{|\mathbf{k}|}\right)+\varepsilon|\mathbf{k}| P_{j}^{(1)}\left(\frac{\mathrm{k}}{\left|\frac{\mathrm{k}}{\mathrm{k}}\right|}\right)+\varepsilon^{2}|\mathbf{k}|^{2} P_{j}^{(2)}(\mathrm{k} \mathbf{k}), \quad|\mathrm{k}| \rightarrow 0, \quad \mathrm{k} \neq 0,
$$

For each fixed $k$ the operators $P_{j}^{(0)}(\mathbb{k})$ are orthogonal projections on $L_{2}\left(\mathbb{R}^{3}\right)$;

$$
P^{(0)}=\sum_{j=0}^{4} P_{j}^{(0)}\left(\frac{\mathrm{k}}{|\mathbf{k}|}\right)
$$

is the orthogonal projection onto $\mathcal{N}^{(0)}=\operatorname{lin}\left\{\omega^{\frac{1}{2}} \phi_{l}: l=0, \ldots, 4\right\}$ in $L_{2}\left(\mathbb{R}^{3}\right), \phi_{l}$ are given by $(2.3) ; P^{(0)}$ is independent of $\frac{\mathbf{k}}{|\mathbf{k}|}$; For each fixed $\alpha$, the operator $P_{j}^{(l)}$ satisfies (for $l=0,1,2$ and $j=0, \ldots, 4$ )

$$
\left\|P_{j}^{(l)} g\right\|^{(\alpha)} \leq c\|g\|^{\left(\alpha^{\prime}\right)}, \quad \forall \alpha^{\prime}>\frac{3}{2} ;
$$

The operator $U_{\varepsilon}$ can be decomposed

$$
U_{\varepsilon}(t, \mathbf{k})=e^{t \widehat{A}_{\varepsilon}(\mathbf{k})}+\widetilde{U}_{\varepsilon}(t, \mathbf{k}),
$$


where $\widehat{A}_{\varepsilon}(\mathbb{k})=-\left(i \mathbb{k} \cdot \mathbb{v}+\frac{1}{\varepsilon} \nu(\mathbb{v})\right)$, and for each fixed $\alpha>\frac{5}{2}$ the operator $\widetilde{U}_{\varepsilon}$ satisfies

$$
\left\|\widetilde{U}_{\varepsilon}(t, \mathbb{K}) g\right\|^{(\alpha)} \leq c \exp \left(-\sigma \frac{t}{\varepsilon}\right)\|g\|^{(\alpha-1)},
$$

for some $\sigma>0$.

Put $P^{(0) \perp}=1-P^{(0)}$ and note that

$$
\begin{gathered}
P^{(0) \perp} P_{j}^{(0)}=0, \quad P^{(0) \perp} P_{j}^{(1)} P^{(0) \perp}=0, \quad j=0, \ldots, 4 ; \\
P^{(0)} L=L P^{(0)}=0, \quad L=P^{(0) \perp} L=L P^{(0) \perp} .
\end{gathered}
$$

The following elementary inequalities are needed throughout the paper (the proofs are straightforward)

Lermama 3.1.

(i) For any $x \in \mathbb{R}^{1}-\{0\}$ and $\delta \in[0,1]$

$$
\left|\frac{e^{2 x}-1}{x}\right| \leq c_{\delta}|x|^{\delta-1}
$$

(ii) For any $x \in \mathbb{R}^{1}-\{0\}$ and $\delta \in[0,1]$

$$
\left|\frac{e^{i x}-1}{i x}-1\right| \leq c_{\delta}|x|^{\delta}
$$

(iii) For any $\beta_{1}>3$ and any $\beta_{2}>3$

$$
\int_{\mathbb{R}_{3}} \frac{1}{\left(1+\left|\mathbb{R}_{1}\right|\right)^{\beta_{1}}\left(1+\left|\mathbb{k}-\mathbb{k}_{1}\right|\right)^{\beta_{2}}} d \mathbb{k}_{1} \leq \frac{c}{(1+|\mathbb{k}|)^{\beta_{1} \wedge \beta_{2}}} ;
$$

(iv) For $\xi, \gamma, \eta>0$

$$
\int_{0}^{t} \exp \left(-\xi\left(t-t_{1}\right)-\left(\gamma-\eta t_{1}\right)|\mathbb{k}|\right) \mathrm{d} i_{1} \leq \frac{1}{\xi+\eta|\mathbb{k}|} \exp (-(\gamma-\eta t)|\mathbb{k}|)
$$

One has

Lemmana 3.2 ([14])。 Let $\alpha>\frac{3}{2}$ and $G \in \dot{X}$. Then

(i) $e^{i B_{e}} G \in \mathbb{Z}$;

(ii) $\left\|e^{t B_{*}} G\right\|_{\mathbb{Z}} \leq c\|G\|_{\mathbb{X}}$.

Define the operators $\mathfrak{A}_{\varepsilon}$ and $\mathfrak{B}_{\varepsilon}$ by 


$$
\mathfrak{A}_{\varepsilon} g(t)=\int_{0}^{t} e^{\left(t-t_{1}\right) B_{\varepsilon}} P^{(0) \perp} g\left(t_{1}\right) \mathrm{d} t_{1}
$$

and

$$
\mathfrak{B}_{\varepsilon} g(t)=\int_{0}^{t} e^{\left(t-t_{1}\right) B_{\varepsilon}} g\left(t_{1}\right) \mathrm{d} t_{1} .
$$

The nonlinear operator $\Gamma_{\varepsilon}$ can be decomposed as follows

$$
\Gamma_{\varepsilon}\left(g_{1}, g_{2}\right)=\Gamma_{0}\left(g_{1}, g_{2}\right)+\Lambda_{\varepsilon}\left(g_{1}, g_{2}\right) \text {, }
$$

where

$$
\begin{aligned}
& \Gamma_{0}\left(g_{1}, g_{2}\right)(t, \mathbf{x}, \mathbf{v})=\frac{1}{2} \int_{\mathbb{R}^{3}} \int_{\mathbb{S}^{2}}\left(g_{1}\left(t, \mathbf{x}, \mathbf{w}^{\prime}\right) g_{2}\left(t, \mathbf{x}, \mathbf{v}^{\prime}\right)+g_{1}\left(t, \mathbf{x}, \mathbf{w}^{\prime}\right) g_{2}\left(t, \mathbf{x}, \mathbf{w}^{\prime}\right)-\right. \\
& \left.g_{1}(t, \mathbf{x}, \mathbf{w}) g_{2}(t, \mathbf{x}, \mathbf{v})-g_{1}(t, \mathbf{x}, \mathbf{v}) g_{2}(t, \mathbf{x}, \mathbf{w})\right) \omega^{\frac{1}{2}}(\mathbf{w})(\mathbb{R} \cdot(\mathbb{w}-\mathbf{v}) \vee 0) \mathrm{d} \mathbf{n} \mathrm{d} \mathbf{w}
\end{aligned}
$$

and

$$
\begin{aligned}
& \Lambda_{\varepsilon}\left(g_{1}, g_{2}\right)(t, \mathbf{x}, \mathbf{v})=\frac{1}{2} \iint_{\mathbb{R}^{3}} \int_{\mathbb{S}^{2}}\left(\left(g_{1}\left(t, \mathbf{x}+b \varepsilon \mathbf{n}, \mathbf{w}^{\prime}\right)-g_{1}\left(t, \mathbf{x}, \mathbf{w}^{\prime}\right)\right) g_{2}\left(t, \mathbf{x}, \mathbf{w}^{\prime}\right)+\right. \\
& g_{1}\left(t, \mathbf{x}, \mathbf{v}^{\prime}\right)\left(g_{2}\left(t, \mathbf{x}+b \varepsilon \mathbb{R}, \mathbf{w}^{\prime}\right)-g_{2}\left(t, \mathbf{x}, \mathbf{w}^{\prime}\right)\right)- \\
& \left(g_{1}(t, \mathbf{x}-b \varepsilon \mathbf{R}, \mathbf{w})-g_{1}(t, \mathbf{x}, \mathbf{w})\right) g_{2}(t, \mathbf{x}, \mathbf{v})- \\
& g_{1}(t, \mathbf{x}, \mathbf{v})\left(g_{2}(t, \mathbf{x}-b \varepsilon \mathbf{n}, \mathbf{w})-g_{2}(t, \mathbf{x}, \mathbf{w})\right) \omega^{\frac{1}{2}}(\mathbf{w})(\mathbf{p} \cdot(\mathbf{w}-\mathbf{v}) \vee 0) \mathrm{d} \mathbf{n d} \mathbf{w}
\end{aligned}
$$

The operator $\Gamma_{0}$ satisfies $([5,9])$

$$
P^{(0)} \Gamma_{0}\left(g_{1}, g_{2}\right)=0,
$$

for $g_{1}$ and $g_{2}$ such that the integrals make sense.

Ukai and Asano proved

Lemma 3.3 ([14]). Let $\alpha>\frac{5}{2}, \beta>3$ and $g_{1}, g_{2} \in \mathbb{Z}$. Then

(i) $\frac{1}{\varepsilon} \mathfrak{U}_{\varepsilon} \Gamma_{0}\left(g_{1}, g_{2}\right) \in \mathbb{Z}$ :

(ii) $\left\|\frac{1}{\varepsilon} \mathfrak{A}_{\varepsilon} \Gamma_{0}\left(g_{1}, g_{2}\right)\right\|_{\mathbb{Z}} \leq c\left(1+\frac{1}{\eta}\right)\left\|g_{1}\right\|_{\mathbb{Z}}\left\|g_{2}\right\|_{\mathbb{Z}}$.

On the other hand, the operator $\Lambda_{\varepsilon}$ lacks the property like (3.15), but is such that 
Hemrama 3.4. Let $\alpha>\frac{5}{2}, \beta>4$ and $g_{1}, g_{2} \in \mathbb{Z}$. Then

(i) $\frac{1}{\varepsilon} \mathfrak{B}_{\varepsilon} \Lambda_{\varepsilon}\left(g_{1}, g_{2}\right) \in \mathbb{Z}$;

(ii) $\left\|\frac{1}{\varepsilon} \mathfrak{B}_{\varepsilon} \Lambda_{\varepsilon}\left(g_{1}, g_{2}\right)\right\|_{\mathbb{Z}} \leq c\left(1+b+\frac{b}{\eta}\right)\left\|g_{1}\right\|_{\mathbb{Z}}\left\|g_{2}\right\|_{\mathbb{Z}}$.

Proof. First we prove (ii). One has

$\mathscr{F} \mathbb{A}_{\varepsilon}\left(g_{1}, g_{2}\right)(t, \mathbb{k}, \mathbb{v})=$

$\frac{(2 \pi)^{\frac{3}{2}}}{2} \int_{\mathbb{R}^{3}} \int_{\mathbb{R}^{3}} \int_{\mathbb{S}^{2}}\left(\left(\exp \left(i b \varepsilon \mathbb{R} \cdot \mathbb{k}_{1}\right)-1\right)\left(\widehat{g}_{1}\left(t, \mathbb{k}_{1}, \mathbb{w}^{\prime}\right) \widehat{g}_{2}\left(t, \mathbb{k}-\mathbb{k}_{1}, v^{\prime}\right)+\right.\right.$

$\left.\widehat{g}_{1}\left(t, \mathbb{R}-\mathbb{k}_{1}, \mathbb{v}^{\prime}\right) \widehat{g}_{2}\left(t, \mathbb{k}_{1}, \mathbb{W}^{\prime}\right)\right)-\left(\exp \left(-i b \in \mathbb{R} \cdot \mathbb{R}_{1}\right)-1\right)\left(\widehat{g}_{1}\left(t, \mathbb{k}_{1}, \mathbb{W}\right) \widehat{g}_{2}\left(t, \mathbb{k}-\mathbb{R}_{1}, \mathbb{V}\right)+\right.$ $\left.\left.\widehat{g}_{1}\left(t, \mathbb{R}-\mathbb{K}_{1}, \mathbb{v}\right) \widehat{g}_{2}\left(t, \mathbb{R}_{1}, \mathbb{W}\right)\right)\right) \omega^{\frac{1}{2}}(\mathbb{w})(\mathbb{R} \cdot(\mathbb{y}-\mathbb{V}) \vee 0) \mathrm{d} n d \mathbb{w}_{\mathrm{d}} \mathrm{d} \mathbb{R}_{1}$.

Hence, using Grad's estimates ([9]) and Lemma 3.1(i),

$$
\begin{aligned}
& \left\|\frac{1}{\nu} \mathscr{F} \Lambda_{\varepsilon}\left(g_{1}, g_{2}\right)\left(t, \mathbb{k},{ }^{\circ}\right)\right\|^{(\alpha)} \leq \\
& c b^{\delta} \varepsilon^{\delta}\left\|g_{1}\right\|_{\mathbb{Z}} \mid g_{2} \|_{\mathbb{Z}} \int_{\mathbb{R}^{j}} \frac{\left|\mathbb{k}_{1}\right|^{\delta} \exp \left(-(\gamma-\eta t)\left(\left|\mathbb{K}_{\mathbb{R}}-\mathbb{k}_{1}\right|+\left|\mathbb{k}_{1}\right|\right)\right)}{\left(1+\left|\mathbb{R}_{1}\right|\right)^{\beta}\left(1+\left|\mathbb{R}_{\mathbb{K}}-\mathbb{k}_{1}\right|\right)^{\beta}} \mathrm{d} \mathbb{k}_{\mathbb{1}},
\end{aligned}
$$

for $\alpha \geq 1, \delta \in[0,1], t \in\left[0, t_{0}\right], \mathbb{k} \in \mathbb{R}^{3}$.

Then, by Lemma 3.1 (iii),

$$
\left\|\frac{1}{\nu} \mathscr{F}_{\Lambda_{\varepsilon}}\left(g_{1}, g_{2}\right)\left(t, \mathbb{k},{ }^{o}\right)\right\|^{(\alpha)} \leq \frac{c b^{\delta} \varepsilon^{\delta}}{(1+|\mathbb{k}|)^{\beta-\delta} \exp ((\gamma-\eta t)|\mathbb{K}|)}\left\|g_{1}\right\|\left\|_{\mathbb{Z}} g_{2}\right\|_{\mathbb{Z}},
$$

provided that $\beta>3+\delta$.

One can now proceed analogously to the proof of Lemma 3.3 (cf. Proposition 2.6 in [14]).

Combining $(3.7),(3.9),(3.4 \mathrm{~b}),(3.16)$ (with $\delta=1$ ) and Lemma 3.1 (iv) (with $\xi=0$ ) yields

$$
\begin{aligned}
& \left\|\frac{1}{\varepsilon} \int_{0}^{t} \chi(\varepsilon|\mathbb{k}| \leq \kappa) \sum_{j=0}^{4} e^{\lambda_{j}^{\xi}(\mathbf{k})\left(t-t_{1}\right)} P_{j}^{\varepsilon}(\mathbb{k}) \mathscr{F} \Lambda_{\varepsilon}\left(g_{1}, g_{2}\right)(t, \mathbb{k}, \cdot) \mathrm{d} t_{1}\right\|(\boldsymbol{\alpha}) \leq \\
& \frac{c b\left(1+\frac{1}{\eta}\right)}{(1+|\mathbb{k}|)^{\beta} \exp ((\gamma-\eta t)|\mathbb{k}|)}\left\|g_{1}\right\|_{\mathbb{Z}}\left\|g_{2}\right\|_{\mathbb{Z}} .
\end{aligned}
$$

Similarly, by (3.11), (3.4b), (3.16) (with $\delta=0)$ and Lemma 3.1 (iv) (with $\left.\xi=\frac{\sigma}{\varepsilon}\right)$, 
$\left\|\frac{1}{\varepsilon} \int_{0}^{t} \widetilde{U}_{\varepsilon}\left(t-t_{1}, \mathbf{k}\right) \mathscr{F} \Lambda_{\varepsilon}\left(g_{1}, g_{2}\right)(t, \mathbf{k}, \cdot) \mathrm{d} t_{1}\right\|^{(\alpha)} \leq \frac{c\left\|g_{1}\right\| \mathbb{Z}\left\|g_{2}\right\| \mathbb{Z}}{(1+|\mathbf{k}|)^{\beta} \exp ((\gamma-\eta t)|\mathbb{k}|)}$

Finally, by Lemma 3.1 (iv) (with $\left.\xi=\frac{\nu}{\varepsilon}\right)$,

$$
\left\|\frac{1}{\varepsilon} \int_{0}^{t} e^{\left(t-t_{1}\right) \tilde{A}_{(}(\mathbf{k})} \mathscr{F} \Lambda_{\varepsilon}\left(g_{1}, g_{2}\right)\left(t_{1}, \mathbf{k}, \cdot\right) \mathrm{d} t_{1}\right\|^{(\alpha)} \leq \frac{c\left\|g_{1}\right\| z_{Z}\left\|g_{2}\right\|}{(1+|\mathbf{k}|)^{\beta} \exp ((\gamma-\eta t)|\mathbf{k}|)} .
$$

Hence (ii) is obtained.

We will have established the lemma if we prove (i). But this follows in much the same way as Lemma 3.3(i).

A similar consideration can be applied in order to prove

Lemma 3.5. Let $\alpha>\frac{5}{2}, \beta \geq 0$ and $g \in \mathbb{Z}$. Then

(i) $\underline{1}_{\varepsilon} \mathbb{B}_{\varepsilon} Q_{\varepsilon} g \in \mathbb{Z}$

(ii) $\left\|\frac{1}{\varepsilon} \mathfrak{Y}_{\varepsilon} Q_{\varepsilon} g\right\|_{\mathbb{Z}} \leq c b\left(1+\frac{1}{\eta}\right)\|g\|_{\mathbb{Z}}$.

Proof. One has

$$
\begin{aligned}
& \mathscr{F} Q_{\varepsilon} g(t, \mathbf{k}, \mathbf{v})=\iint_{\mathbb{R}^{3}} \int_{\mathbb{S}^{2}}\left((\exp (i b \varepsilon \mathbf{n} \cdot \mathbf{k})-1) \widehat{g}\left(t, \mathbf{k}, \mathbf{w}^{\prime}\right) \omega^{\frac{1}{2}}\left(\mathbf{v}^{\prime}\right)-\right. \\
& \left.(\exp (-i b \mathbf{\varepsilon} \mathbf{n} \cdot \mathbf{k})-1) \widehat{g}(t, \mathbf{k}, \mathbf{w}) \omega^{\frac{1}{2}}(\mathbf{v})\right) \omega^{\frac{1}{2}}(\mathbf{w})(\mathbf{n} \cdot(\mathbf{w}-\mathbf{v}) \vee 0) \mathrm{d} \mathbf{n d w}
\end{aligned}
$$

The same estimation (with respect to the $\nabla$-variable), as that for the operator $K$ defined in $(3.4 \mathrm{a})$ - cf. [9], works for the linear operator $Q_{\varepsilon}$. Therefore, by Lemma $3.1(\mathrm{i})$ (with $\delta=1$ ), one obtains

$$
\left\|\mathscr{F} Q_{\varepsilon} g\left(t, \mathbf{k},{ }^{\circ}\right)\right\|(\alpha) \leq \frac{c b \varepsilon|\mathbf{k}|}{(1+|\mathbf{k}|)^{\beta} \exp ((\gamma-\eta t)|\mathbf{k}|)}\|g\|_{\mathbb{Z} .}
$$

Thus, by (3.5) and Lemma 3.1 (iv) (with $\xi=0$ ), (ii) follows. Then (i) is standard.

By (3.14) and (3.15), Problem (3.3) can be rewritten in the form

$$
g(t)=e^{i B_{\varepsilon}} G+\frac{1}{\varepsilon} \mathfrak{B}_{\varepsilon} Q_{\varepsilon} g(t)+\frac{1}{\varepsilon} \mathfrak{A}_{\varepsilon} \Gamma_{0}(g, g)(t)+\frac{1}{\varepsilon} \mathfrak{V}_{\varepsilon} \Lambda_{\varepsilon}(g, g)(t)
$$

For fixed $G$, let $\mathfrak{N}_{\varepsilon}(g)(t)$ be defined by the right-hand side of Eq. (3.22). 
Combining Lemmas 3.1-3.5 yields

$$
\left\|\mathfrak{R}_{\varepsilon}(g)\right\|_{\mathbb{Z}} \leq \mathfrak{c}_{1}\|G\|_{\mathbb{Z}}+\mathfrak{c}_{2} b\left(1+\frac{1}{\eta}\right)\|g\|_{\mathbb{Z}}+\mathfrak{c}_{3}\left(1+b+\frac{b}{\eta}\right)\|g\|_{\mathbb{Z}}^{2}
$$

as well as

$$
\left\|\mathfrak{N}_{\varepsilon}\left(g_{1}\right)-\mathfrak{R}_{\varepsilon}\left(g_{2}\right)\right\|_{\mathbb{Z}} \leq\left(\mathfrak{c}_{2} b\left(1+\frac{1}{\eta}\right)+\mathfrak{c}_{3}\left(1+b+\frac{b}{\eta}\right)\left\|g_{1}+g_{2}\right\|_{\mathbb{Z}}\right)\left\|g_{1}-g_{2}\right\|_{\mathbb{Z}},
$$

where the constants $c_{1}, c_{2}$ and $c_{3}$ are independent of $b, \eta$ and $t_{0}$.

Let now $\alpha, \beta$ and $\gamma$ be such that

$$
\alpha>\frac{5}{2}, \quad \beta>4, \quad \gamma>0
$$

moreover let $b, \eta$ and $t_{0}$ be fixed such that

$$
\begin{gathered}
0<b<\frac{1}{\mathfrak{c}_{2}}, \\
\eta>\frac{\mathfrak{c}_{2} b}{1-\mathfrak{c}_{2} b}>0
\end{gathered}
$$

and

$$
t_{0}=\frac{r}{\eta}
$$

Assuming that

$$
\|G\|_{\mathbb{X}}<\vartheta_{0} \equiv \frac{\left(1-\mathfrak{c}_{2} b\left(1+\frac{1}{\eta}\right)\right)^{2}}{4 \mathfrak{c}_{1} \mathfrak{c}_{3}\left(1+b+\frac{b}{\eta}\right)}
$$

one has

$$
g \in \mathbb{Z}_{0} \Leftrightarrow \mathfrak{N}_{\varepsilon}(g) \in \mathbb{Z}_{0}
$$

and

$$
\left\|\mathfrak{N}_{\varepsilon}\left(g_{1}\right)-\mathfrak{N}_{\varepsilon}\left(g_{2}\right)\right\|_{\mathbb{Z}} \leq \vartheta_{1}\left\|g_{1}-g_{2}\right\|_{\mathbb{Z}} \quad \forall g_{1}, g_{2} \in \mathbb{Z}_{0}
$$

where

$$
\mathbb{Z}_{0}=\left\{g \in \mathbb{Z}: \quad\|g\|_{\mathbb{Z}} \leq \vartheta_{2}\right\}
$$

with

$$
\left.\vartheta_{1}=1-\left(\left(1-\mathfrak{c}_{2} b\left(1+\frac{1}{\eta}\right)\right)^{2}-4 \mathfrak{c}_{1} \mathfrak{c}_{3}\left(1+b+\frac{b}{\eta}\right)\|G\|_{\mathbb{X}}\right)^{\frac{1}{2}} \in\right] 0,1[
$$


and

$$
\vartheta_{2}=\frac{\vartheta_{1}-c_{2} b\left(1+\frac{1}{\eta}\right)}{2 c_{3}\left(1+b+\frac{b}{\eta}\right)} \geq 0
$$

Hence $\mathfrak{R}_{\varepsilon}$ is a contracting operator on $\mathbb{Z}_{0}$ and therefore it has a unique fixed point in $\mathbb{Z}_{0}$. This is the unique solution of the integral equation (3.3). Actually this solution is a unique classical solution of Problem (3.2) as the arguments of Ukai and Asano [14] show. Moreover, it follows, by these arguments, that the solution is continuously differentiable in $\mathbb{Y}_{\beta-1, r}^{\alpha-1, \eta}\left(\left[0, t_{0}\right]\right)$. Summarizing, one has

Theorem 3.1. Let $\alpha, \beta, \gamma, b, \eta$ and $t_{0}$ be such that (3.25-26) hold. If the initial datum $G \in \dot{X}_{\beta, r}^{(\alpha)}$ satisfies the smallness condition (3.27), then there exists a unique classical solution $g$ of Problem (3.2) on the time interval $\left[0, t_{0}\right]$ such that

$$
\begin{gathered}
g \in \mathbb{Z}_{\beta, 7, t_{0},}^{\alpha, \eta, 1},\|g\|_{\beta, r, t_{0}}^{\alpha, \eta, 1} \leq \vartheta_{2}, \\
\left.\left.\frac{\partial}{\partial t} g \in C^{0}(] 0,1\right] ; \quad \mathbb{Y}_{\beta-1, \gamma}^{\alpha-1, \eta}\left(\left[0, t_{0}\right]\right)\right) .
\end{gathered}
$$

\section{$\S 4$. Hydrodynamic limit}

Still following [14], consider the subspace $\mathbb{W}=\mathbb{W}_{\beta, r, t_{0}}^{\alpha, \eta, 1}$ of the space $\mathbb{Z}=\mathbb{Z}_{\beta, r, t_{0}}^{\alpha, \eta, 1}$ defined as

$$
\left.\left.\mathbb{W}=\left\{g \in \mathbb{Z}: \quad \exists g_{0} \in \mathbb{Y}(] 0, t_{0}\right]\right), \quad \forall \delta>0, \quad\left\|g-g_{0}\right\|_{\mathbb{Y}\left(\left[\delta . t_{0}\right]\right)} \rightarrow 0 \text { as } \varepsilon \downarrow 0\right\}
$$

(the numbers $\alpha, \beta, \gamma, b, \eta$ and $t_{0}$ are chosen such that (3.25-26) are satisfied).

The following two lemmas were proved by Ukai and Asano

Lemma 4.1 ( $[14])$. Let $G \in \dot{X}$. Then $e^{t B_{c}} G \in \mathbb{W}$ with the limit $E(t) G$, where $E(t)$ is given by

$$
\left.\left.\mathscr{F} E(t)=\sum_{j=0}^{4} \exp \left(i \lambda_{j}^{(1)}|\mathbf{k}| t\right) P_{j}^{(0)}\left(\frac{\mathbf{k}}{|\mathbf{k}|}\right), \quad t \in\right] 0, t_{0}\right] .
$$

Lemma 4.2 ([14]). Let $g \in \mathbb{W}$ with the limit $g_{0}$. Then $\mathfrak{A}_{\varepsilon} \Gamma_{0}(g, g) \in \mathbb{W}$ with the limit $\widetilde{\mathfrak{U}}_{0} \Gamma_{0}\left(g_{0}, g_{0}\right)$, where

$$
\mathscr{F} \widetilde{\mathfrak{U}}_{0} \Gamma_{0}\left(g_{0}, g_{0}\right)(t, \mathbf{k}, \cdot)=
$$


$\sum_{j=0}^{4} \int_{0}^{t} \exp \left(i \lambda_{j}^{(1)}|\mathrm{K}|\left(t-t_{1}\right)\right)|\mathrm{d}| P_{j}^{(1)}\left(\frac{\mathrm{K}}{|\mathrm{K}|}\right) \mathscr{F} \Gamma_{0}\left(g_{0}, g_{0}\right)\left(t_{1}\right) \mathrm{d} t_{1}-L^{-1} \mathscr{F}_{F} \Gamma_{0}\left(g_{0}, g_{0}\right)(t)$, for $\left.t \in] 0, t_{0}\right]$.

It remains to prove

Lemmma 4.3. Let $g \in \mathbb{W}$ with the limit $g_{0}$. Then $\frac{1}{\varepsilon} \mathfrak{B}_{\varepsilon} \Lambda_{\varepsilon}(g, g) \in \mathbb{W}$ with the limit $\widetilde{\mathfrak{S}}_{0} \widetilde{\Lambda}_{0}\left(g_{0}, g_{0}\right)$, where

$$
\widetilde{\mathfrak{S}}_{0} f(t)=\int_{0}^{t} E\left(t-t_{1}\right) f\left(t_{1}\right) \mathrm{d} t_{1}
$$

and

$$
\begin{aligned}
& \mathscr{F} \widetilde{\Lambda}_{0}\left(g_{0}, g_{0}\right)(t, \mathbb{R}, \mathbb{v})=\frac{(2 \pi)^{\frac{3}{2}}}{2} i b \iint_{\mathbb{R}^{3}} \int_{\mathbb{R}^{3}} \int_{\mathbb{S}^{2}} \cdot \mathbb{k}_{1}\left(\widehat{g}_{0}\left(t, \mathbb{R}_{1}, \mathbb{w}^{\prime}\right) \widehat{g}_{0}\left(t, \mathbb{k}-\mathbb{k}_{1}, \mathbb{v}^{\prime}\right)+\right.
\end{aligned}
$$

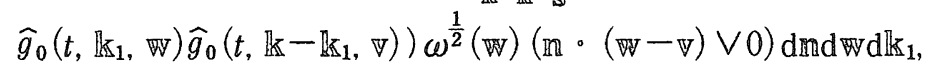

Proof. Repeating the argument of the proof of Lemma 4.1 (cf. [14] Proposition 3.1) and applying (3.17) with $\delta=1$, as well as Lemma 3.1 (iv) with $\xi=0$ yield

$$
\begin{aligned}
& \| \chi\left(|\mathbb{R}| \leq \frac{\kappa}{\varepsilon^{\frac{1}{4}}}\right) \frac{1}{\varepsilon} \int_{0}^{t} \sum_{j=0}^{4}\left(e^{\lambda f(\mathbb{k})\left(t-t_{1}\right)} P_{j}^{\varepsilon}(\mathbb{k})-e^{2 \lambda_{j}^{(1)}(\mathbf{k})\left(t-t_{1}\right)} P_{j}^{(0)}\left(\frac{\mathbb{k}}{|\mathbb{k}|}\right)\right) \\
& \mathscr{F} \Lambda_{\varepsilon}(g, g) \mathrm{d} t_{1}\left\|_{\mathbb{Y}\left(\left[0 . t_{0}\right]\right)} \leq c b \varepsilon^{\frac{1}{2}}\left(1+\frac{1}{\eta}\right)\right\| g \|_{\mathbb{Z}}^{2} .
\end{aligned}
$$

By $(3.10),(3.11),(3.16)$ with $\delta=1$ and Lemma 3.1 (iv), one has

$$
\left\|\chi\left(|\mathbb{Z}| \leq \frac{\kappa}{\varepsilon^{\frac{1}{4}}}\right) \frac{1}{\varepsilon} \int_{0}^{t} U_{\varepsilon}\left(t-t_{1}, \mathbb{k}\right) \mathscr{F} \Lambda_{\varepsilon}(g, g) \mathrm{d} t_{1}\right\|_{\mathbb{Y}\left(\left[0, t_{0}\right]\right)} \leq c b \varepsilon^{\frac{3}{4}} \leq\|g\|_{\mathbb{Z}}^{2} .
$$

Let $\delta \in] 0, t_{0}$ [ be fixed. Proceeding analogously to the proof of Lemma 3.4, and applying the argument of Lemma 3.2 in [14] give

$$
\begin{aligned}
& \left\|\chi\left(\left|\mathbb{K}_{\mathbb{K}}\right| \leq \frac{\kappa}{\varepsilon^{\frac{1}{4}}}\right) \frac{1}{\varepsilon}\left(\mathfrak{B}_{\varepsilon}-\widetilde{\mathfrak{B}}_{0}\right) \Lambda_{\varepsilon}(g, g)\right\|_{\mathbb{Y}\left(\left[\delta, t_{0}\right]\right)} \leq \\
& c\left(1+b+\frac{b}{\eta}\right)\|g\|_{\mathbb{Z}}\left\|\chi\left(|\mathbb{R}| \leq \frac{\kappa}{2 \varepsilon^{\frac{1}{4}}}\right) g\right\|_{\mathbb{Y}\left(\left[\tilde{\delta}_{1}, t_{0}\right]\right)}+c\left(1+\frac{b}{\eta}\right) \frac{\delta_{1}}{\delta-\delta_{1}}\|g\|_{\mathbb{Z}}^{2},
\end{aligned}
$$


for each $\left.\delta_{1} \in\right] 0, \delta[$.

Since $g \in W$, both terms in the right-hand side of (4.3) may be made arbitrarily small by choosing $\delta_{1}$ and $\varepsilon$ to be small enough. Therefore, (4.1), (4.2) and (4.3) yield

$$
\left\|\frac{1}{\varepsilon}\left(\mathfrak{B}_{\varepsilon}-\widetilde{\mathfrak{B}}_{0}\right) \Lambda_{\varepsilon}(g, g)\right\|_{\mathbb{Y}\left(\left[\delta, t_{0}\right]\right)} \rightarrow 0 \quad \text { as } \quad \varepsilon \downarrow 0,
$$

for each $\delta \in] 0, t_{0}[$.

Similarly, applying Lemma 3.1 (ii) with $\delta=1\left(\right.$ for $\left.|\mathbf{k}| \leq \frac{1}{\varepsilon^{\frac{1}{4}}}\right)$ and with $\delta=0$ (for $\left.|\mathbf{k}| \geq \frac{1}{\varepsilon^{\frac{1}{4}}}\right)$, one obtains

$$
\left\|\tilde{\mathfrak{B}}_{0}\left(\frac{1}{\varepsilon} \Lambda_{\varepsilon}(g, g)-\tilde{\Lambda}_{0}(g, g)\right)\right\|_{\mathbb{Y}\left(\left[\delta, t_{\epsilon}\right]\right)} \rightarrow 0 \quad \text { as } \quad \varepsilon \downarrow 0 .
$$

for each $\delta \in] 0, t_{0}[$.

Finally, as in the proof of Lemma 4.2 (cf. [14]), one has

$$
\left\|\widetilde{\mathfrak{B}}_{0}\left(\widetilde{\Lambda}_{0}(g, g)-\widetilde{\Lambda}_{0}\left(g_{0}, g_{0}\right)\right)\right\|_{\mathbb{Y}\left(\left[\delta, t_{0}\right]\right)} \rightarrow 0 \quad \text { as } \quad \varepsilon \downarrow 0,
$$

for each $\delta \in] 0, t_{0}[$.

Combining (4.4), (4.5), (4.6) completes the proof.

In the same manner one can prove

Lemma 4.4. Let $g \in \mathbb{W}$ with the limit $g_{0}$. Then $\frac{1}{\varepsilon} \mathfrak{B}_{\varepsilon} Q_{\varepsilon} g \in \mathbb{W}$ with the limit $\widetilde{\mathfrak{S}}_{0} \widetilde{Q}_{0} g_{0}$, where

$$
\begin{aligned}
& \mathscr{F}_{Q_{0}} g_{0}(t, \mathbf{k}, \mathbf{v})= \\
& i b \int_{\mathbb{R}^{3} \mathbb{S}^{2}} \int_{\mathbf{n}} \cdot \mathbb{k}\left(\omega^{\frac{1}{2}}\left(\mathbf{v}^{\prime}\right) \widehat{g}_{0}\left(t, \mathbb{k}, \mathbf{w}^{\prime}\right)+\omega^{\frac{1}{2}}(\mathbf{v}) \widehat{g}_{0}(t, \mathbf{k}, \mathbf{w})\right) \omega^{\frac{1}{2}}(\mathbf{w})(\mathbf{n} \cdot(\mathbf{w}-\mathbf{v}) \vee 0) \mathrm{d} \mathbf{n d w},
\end{aligned}
$$

for $\left.t \in] 0, t_{0}\right]$.

Combining these lemmas, one concludes, that $\mathfrak{N}_{\varepsilon}$ maps $\mathbb{W}$ into itself and therefore $\mathfrak{R}_{\varepsilon}$ is a contraction in $\mathbb{W} \cap \mathbb{Z}_{0}$ (provided Conditions (3.25-27) are satisfied). Hence the solution $g$ of Theorem 3.1 is in WV and $g \rightarrow g_{0}$ as $\varepsilon \downarrow 0$, in the space $\mathbb{Y}\left(\left[\delta, t_{0}\right]\right)$, for any $\left.\left.\delta \in\right] 0, t_{0}\right]$. The limit $g_{0}$ satisfies

$$
g_{0}(t)=E(t) G+\widetilde{\mathfrak{B}}_{0} \widetilde{Q}_{0} g_{0}(t)+\widetilde{\mathscr{U}}_{0} \Gamma_{0}\left(g_{0}, g_{0}\right)(t)+\widetilde{\mathfrak{B}}_{0} \widetilde{\Lambda}_{0}\left(g_{0}, g_{0}\right)(t),
$$

on $\left.] 0, t_{0}\right]$. One can apply the contracting mapping principle in $\mathbb{Y}\left(\left[0, t_{0}\right]\right)$ to $(4.7)$ in order to see that $(4.7)$ is satisfied on $\left[0, t_{0}\right]$ and $g_{0} \in \mathbb{Y}\left(\left[0, t_{0}\right]\right)$.

From (3.12) one has 


$$
P^{(0) \perp} E(t) G=P^{(0) \perp}{\widetilde{\mathfrak{F}_{0}}}_{0} \widetilde{Q}_{0} g_{0}(t)=P^{(0) \perp \widetilde{\mathfrak{B}}_{0}} \widetilde{\Lambda}_{0}\left(g_{0}, g_{0}\right)(t)=0
$$

and from (3.12), (3.15) it follows that

$$
P^{(0) \perp} \widetilde{Q}_{0} \Gamma_{0}\left(g_{0}, g_{0}\right)=-P^{(0) \perp} L^{-1} \Gamma_{0}\left(g_{0}, g_{0}\right) .
$$

Hence, the limit $g_{0}$ satisfies

$$
P^{(0) \perp} g_{0}=-P^{(0) \perp} L^{-1} \Gamma_{0}\left(g_{0}, g_{0}\right),
$$

and by (3.13)

$$
L g_{0}+\Gamma_{0}\left(g_{0}, g_{0}\right)=0
$$

Therefore $f_{0}=\omega+\omega^{\frac{1}{2}} g_{0}$ satisfies Eq. (2.2) and thus is a Maxwellian

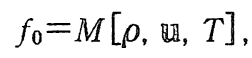

for some fluid-dynamic parameters $\rho$, 재, $T$.

On the other hand, by $(4.7)$, one has

$$
P^{(0)} g_{0}(0)=P^{(0)} G \text {. }
$$

The solution $f$ of Problem (2.1), given by (3.1b), satisfies

$$
\left(\phi_{j}, D f\right)_{L_{2}\left(\mathbb{R}^{3}\right)}=\frac{1}{\varepsilon}\left(\phi_{j}, J_{\varepsilon}(f, f)-J_{0}(f, f)\right)_{L_{2}\left(\mathbb{R}^{3}\right), \quad j=0, \ldots, 4,}
$$

where the notation of Section 2 has been used.

Passing to the limit $\varepsilon \downarrow 0$, one obtains

$$
\begin{aligned}
& \left(\phi_{j}, f_{0}(t)\right)_{L_{2}\left(\mathbb{R}^{3}\right)}-\left(\phi_{j}, F\right)_{L_{2}\left(\mathbb{R}^{3}\right)}= \\
& \int_{0}^{t}\left(-\left(\phi_{j}, \mathrm{v} \cdot \frac{\partial}{\partial \mathrm{X}} f_{0}\left(t_{1}\right)\right)_{L_{2}\left(\mathbb{R}^{3}\right)}+b\left(\psi_{j}, J_{0}^{(1)}\left(f_{0}, f_{0}\right)\left(t_{1}\right)\right)_{L_{2}\left(\mathbb{R}^{3}\right)}\right) \mathrm{d} t_{1},
\end{aligned}
$$

and hence concludes that the parameters $\rho, \mathbb{U}, T$ of $f_{0}$ are a classical solution of the initial-value problem for the Enskog-Euler system (2.9).

Summarizing, one has

Theorem 4.1. Let the hypotheses of Theorem 3.1 be satisfied. Then

(i) $g(t) \rightarrow g_{0}(t)$, as $\varepsilon \downarrow 0$, strongly in $\bigvee_{\beta, \gamma}^{\alpha, \eta}\left(\left[\delta, t_{0}\right]\right)$ for any $\left.\delta \in\right] 0, t_{0}[$;

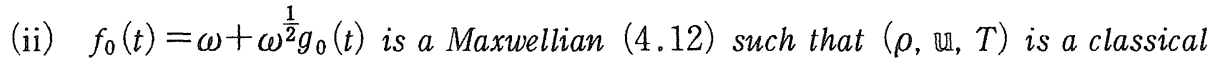
solution of the Enskog-Euler system (2.9) with the initial datum

$$
\begin{gathered}
\left.\rho\right|_{t=0}=\left(\phi_{0}, F\right)_{L_{2}\left(\mathbb{R}^{3}\right),} \\
\left.u_{j}\right|_{t=0}=\left(\phi_{1}, F\right)_{L_{2}\left(\mathbb{R}^{3}\right)}, \quad j=1,2,3,
\end{gathered}
$$




$$
\left.T\right|_{t=0}=\left(\phi_{4}, F\right)_{L_{2}\left(\mathbb{R}_{3}\right)}
$$

Remark 4.1. Theorem 4.1 delivers an existence result for the Enskog-Euler system (2.9). For the Euler system (i.e. for $b=0$ ), which is a symmetric hyperbolic system provided that $\rho>0$, the (local) existence and uniqueness theorem is available for the Cauchy problem with analytical initial data $\left.(\rho, \mathbf{u}, T)\right|_{t=0}$ such that

$$
\left.\rho\right|_{t=0}>0
$$

This assumption was essential in the proof by Nishida [12] of the convergence of solution of the Boltzmann equation to the Maxwellian, which fluid-dynamic parameters solve the Euler system. This type of assumption was also essential in the methods reviewed in the lecture [10]. On the other hand, Assumption (4.17) was removed in methods by Ukai and Asano [14]. Certainly it is necessary neither in this paper. The existence result, which follows from Theorem 4.1, seems to be the first for the Enskog-Euler system.

Remark 4.2. Similarly as for the Boltzmann equation ([14]), when the initial layer vanish, i.e. for

$$
g_{0}=P^{(0)} g_{0},
$$

Theorem 4.1 hold with $\delta=0$. On the other hand the structure of the initial layer can be studied as in paper [3].

Remark 4.3. The reasoning of this paper applies to both cases given in Remarks 2.1 and 2.2, when $b \sim c$. On the other hand, it breaks down for the symmetrized equation, when it results in the Navier-Stokes-type system at the 0 -th order approximation (i.e. for $b \sim c \sqrt{\varepsilon}$, c.f. Remark 2.2).

\section{Acknowledgement}

This work was partially supported by the Polish State Committee for Scientific Research under the Grant 2P03A02311.

\section{References}

[1] Arkeryd, L., On the Enskog equation with large data, SIAM J. Math. Anal., 21 (1990), 631-646.

[2] Arkeryd, L. and Cercignani, C., Global existence in $L^{1}$ for the Enskog equation and convergence of the solutions to solutions of the Boltzmann equation, J. Statist. Phys., $\mathbf{5 9}$ (1990), 3-4. 845-867.

[3] Asano, K. and Ukai, S., On the fluid dymamical limit of the Boltzmann equation, in Lecture Note in Numer. Appl. Anal., Recent Toprcs in Nonlinear PDE 6, Eds. M. Mimura and T. Nishida, 
North-Holland and Kinokuniya 1984, 1-20.

[4] Bellomo, N., Lachowicz, M., Polewczak, J. and Toscani, G., Mathematical Topics in Nonlinear Kinetic Theory II: The Enskog Equation, World Sci., Singapore 1991.

[5] Cercignani, C., Illner, R. and Pulvirenti, M., The Mathematical Theory of Dilute Gases, Springer, New York 1994.

[6] Ellis, R. S. and Pinsky, M. A., The first and second fluid approximations to the linearized Boltzmann equation, J. Math. Pure Appl., 54 (1975), 125-156.

[7] Esteban, M. and Perthame, B., On the modified Enskog equation for elastic and inelastic collisions. Models with spin, Ann. Inst. H. Poincaré Anal. Non Linéaire, 8 (1991), 289-308.

[8] Ferziger, J. H. and Kaper, H. G., Mathematical Theory of Transport Processes in Gases, NorthHolland 1972.

[9] Grad, H., Asymptotic theory of the Boltzmann equation. II, in Rarefied Gas Dynamics, vol. I, Ed. J. Laurmann, Academic Press 1963, 26-59.

[10] Lachowicz, M., Asymptotic analysis of nonlinear kinetic equations: The hydrodynamic limit, in . Lecture Notes on the Mathematical Theory of the Boltzmann equation, World Sci., Singapore 1995, 65-148.

[11] - From kinetic to Navier-Stokes-type equations, Appl. Math. Lett., 10. 5 (1997), 1923.

[12] Nishida, T., Fluid dynamical limit of the nonlinear Boltzmann equation to the level of the compressible Euler equation, Comm. Math. Phys., 61 (1978), 119-148.

[13] Ukai, S., Solutions of the Boltzmann equation, Patterns and Waves-Qualitative Analysis of Nomlinear Differential Equations 18, North-Holland and Kinokuniya, 1986, 18, 37-96.

[14] Ukai, S. and Asano, K.. The Euler limit and initial layer of the nonlinear Boltzmann equation, Hokkaido Math. J., 12, n. 3 (1983), 311-332.

[15] Ukai, S., Point, N. and Ghidouche, H., Sur la solution globale du probleme mixte de l'èquation de Boltzmann non-linèaire, J. Math. Pures Appl., 57 (1978), 203-229. 